\author{
M. Martini ${ }^{1}$, G. Mistrello ${ }^{2}$, S. Amato ${ }^{2}$, M.B. Bilò ${ }^{1}$, S. Agolini ${ }^{1}$, A. Corsi ${ }^{1}$, \\ C. TONTINI ${ }^{3}$, L. ANTONICELLI ${ }^{3}$
}

\title{
Anaphylaxis to baobab fruit: the paradox of "natural healthy food"
}

\author{
${ }^{1}$ Allergy Unit, Department of Clinical and Molecular Sciences, Università Politecnica delle Marche, Ancona, Italy \\ ${ }^{2}$ Lofarma SpA, R and D Department, Milano, Italy \\ ${ }^{3}$ Allergy Unit, Department of Internal Medicine, AOU Ospedali Riuniti, Ancona, Italy
}

\author{
Corresponding author \\ Matteo Martini \\ Allergy Unit \\ Department of Clinical and Molecular Sciences \\ Università Politecnica delle Marche \\ Via Conca 71, 60126, Ancona, Italy \\ Phone: +393474884913 \\ E-mail: matteo.martini.med@gmail.com
}

\section{To the Editor}

Baobab (Adansonia digitata) is a woody plant, characteristic of Africa and North-West Australia, whose fruits and leaves have been largely used by the local African populations as food and in traditional medicine (1-3). The cosmetic and wellness industries are currently promoting the baobab-based products for their claimed moisturizing, non-irritating, and highly penetrating properties, particularly useful in the skin care. Similarly, the food industries, to address the growing consumers' interest in natural / healthy products in the developed countries (e.g. vegan lifestyle), have started a huge trading to import baobab fruit's extracts for their products $(4,5)$. In addition, recent studies have focused on health-promoting properties of the baobab fruit (6) and its possible effect on weight maintenance (7). Consequently, there has been a remarkable increase in the sales of products derived from the baobab fruit. In 2005, the industry of natural products was valued at $\$ 65$ billion / year, with an impressive annual increase of $15-20 \%(4,5)$.

We describe a case of anaphylaxis, few minutes after the ingestion of a snack, in a 31-year-old Caucasian woman who presented at the emergency room for oral pruritus, generalised urticaria, facial angioedema, throat tightness, abdominal pain, and diarrhea. The vital signs were normal, and she was treated with systemic corticosteroids, antihistamines, and saline solution. The clinical history revealed that the woman, willing to start a lifestyle based on vegan foods, tasted for the first time a vegan snack (Lifebar Plus ${ }^{\mathrm{TM}}$ ) at lunch, without any other foods, physical exercise, alcohol, or medications taken before or after. The ingredients reported on the product's label were dates, almonds, dried cherries, raw cashew nuts, baobab fruit pulp, dried cranberry powder, maca powder, and crystal pink himalayan salt, with possible traces of other nuts and sesame. The patient, after the episode, ate all the above-mentioned foods without reactions, with the exception of baobab, maca, and sesame, which were not ingested again. No previous food allergy episodes, comorbidities or concomitant medications were reported, except for a mild rhinitis. We started the allergologic diagnostic work-up two months after the episode. Skin prick testing was performed with food (egg, milk, flour, fish, shrimp, almond, walnut, peanut, hazelnut, peach, tomato, apple, celery, soy, sesame, profilin) and airborne (alternaria, birch, cat, cladosporium, cypress, Dermatophagoides farinae and pteronyssinus, dog, hazel, mugwort, olive, parietaria, penicillium, ragweed, timothy grass) commercial extracts (ALK-Abelló). The whole snack and its ingredients, namely maca and baobab fruit powder, were tested 
by prick-to-prick using raw products. Two healthy subjects were also tested, as negative controls. Singleplex ImmunoCAP (Thermo Fisher Scientific, Uppsala) was used for specific IgE measure of food (almond, cashew nut, date, sesame, omega-5 gliadin) and airborne molecular allergens (Alt a1, Cup a1, Der p1, Der P2, Der p23, Der P10, Par j2, Phl P1, Phl P2, Phl P4, Phl P5, Phl P6, Phl P7, Phl P11, Phl P12, Cyn d1). ELISA and IgE-immunoblot tests were conducted as previously described (8). ELISA inhibition experiments were performed using as inhibitors grass and cypress pollen extracts, or alternaria and house dust mite extracts or peel peach extract. All the extracts were used at two different concentrations ( 30 and $3 \mu \mathrm{g} / \mathrm{ml}$ of extract).

Food skin testing resulted positive only to the whole snack and the baobab fruit $(5 \mathrm{~mm})$, being negative to all the other foods. Skin tests to airborne allergens revealed several sensitizations, consistent with the mild rhinitis (i.e. dust mite, timothy grass, cypress, alternaria, parietaria, dog, and cat). Specific IgE resulted negative for all the tested foods, and the airborne pattern was consistent with the skin results. The direct ELISA test confirmed the IgE reactivity of the patient to the baobab fruit's extract (1307 ODx1000), compared to the negative control (354 ODx1000). IgEs to cross reactive carbohydrate determinants (CCD) were negative. SDS-PAGE profile of the baobab fruit's extract showed the presence of different protein components (figure 1, lane 3); the subsequent IgE-immunoblot analysis evidenced two IgE-binding regions at about 40 and $60 \mathrm{kDa}$ in the patient's serum (figure 1, lane 1). The ELISA inhibition experiments did not show any significant inhibition for all the inhibitors used, taking into account that no increase of inhibition level was observed between 3 and $30 \mu \mathrm{g} / \mathrm{ml}$ inhibitor concentrations. On the contrary, when using the baobab fruit's extract as inhibitor, an inhibition of $92 \%$ was observed (figure 2). Due to the severity of her reaction, the patient refused a food challenge with the baobab fruit. She was discharged with the indication of strict avoidance of all kind of baobab-based products (e.g. foods and cosmetics), and provided with self-injectable adrenaline.

To our knowledge, this is the first case of food allergy to baobab fruit, probably driven by two baobab-specific allergens ( 40 and $60 \mathrm{kDa}$, respectively) that we identified as responsible for a genuine sensitization, leading to anaphylaxis. The study raises some considerations. First of all, prick-to-prick test endorses its diagnostic reliability to identify unknown allergens. Furthermore, the way of sensitization and the paradox of "natural healthy" foods are noteworthy. The IgE-mediated reaction occurred, apparently, without a previous ingestion of baobab. Since the ELISA inhibition results did not reveal cross-reactions, an over-
Figure 1 - SDS-PAGE/IgE-immunoblot: lane 1, patient's serum; lane 2, healthy control serum; lane 3, protein profile of the baobab fruit's extract.

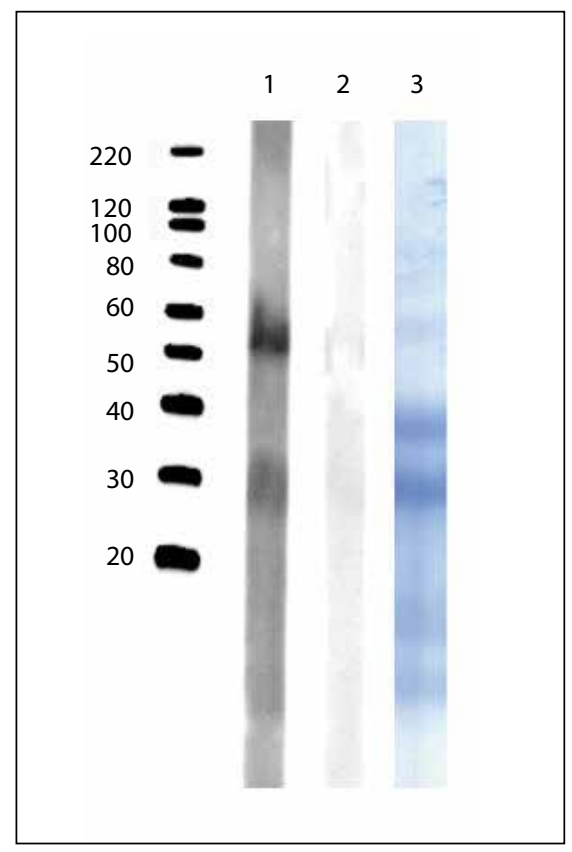

Figure 2 - ELISA inhibition test.

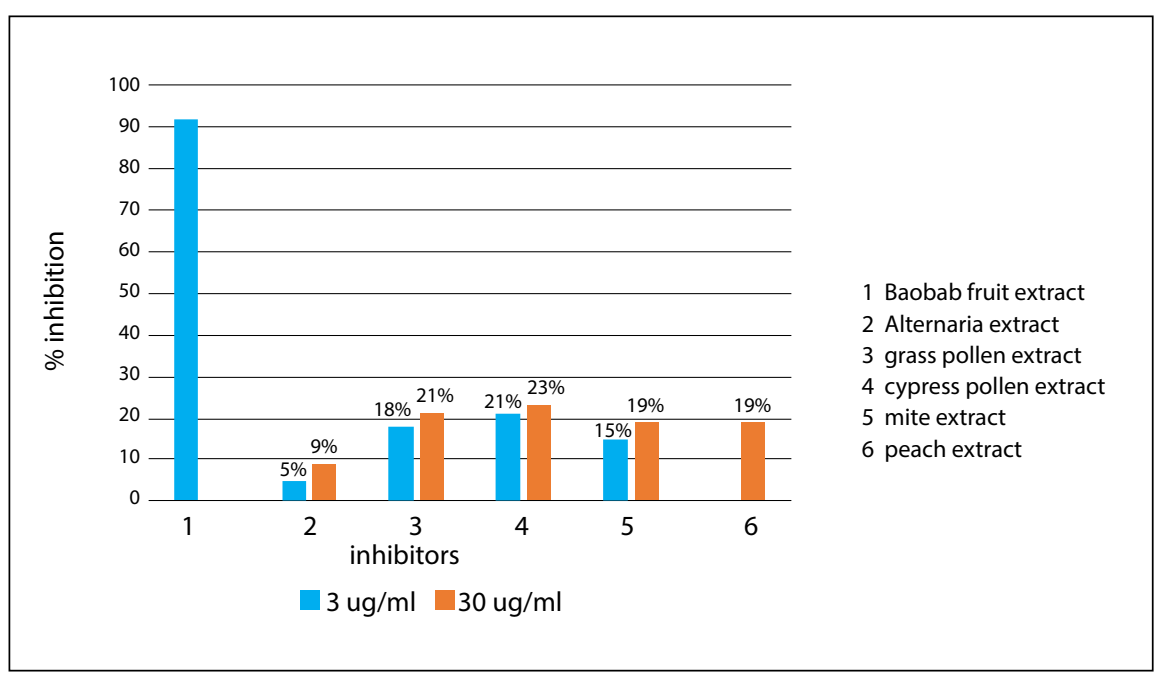


looked previous sensitization to baobab-derived products (i.e. foods or cosmetics (9)), cannot be excluded. The first paradox is related to the myth that natural products cannot be harmful. Another paradox concerns the baobab fruit, that albeit widely used in traditional medicine in the native regions, becomes a dangerous allergen in western countries. Consistently with the model of the peanut allergy (10), the first exposure in adult age and the different processing methods of the baobab fruit in the developed countries, compared to the native countries, could promote its allergenic properties.

In conclusion, the baobab fruit may trigger severe food allergy reactions. Taking into account the increasing market of natural products, similar cases should be expected in the near future.

\section{Conflict of interest}

The authors declare that they have no conflict of interest.

\section{References}

1. Mokganya MG, Tshisikhawe MP: Medicinal uses of selected wild edible vegetables consumed by Vhavenda of the Vhembe District Municipality, South Africa. South African J Bot 2018; DOI:10.1016/J.SAJB.2018.09.029.
2. Sugandha S, Varsha P, Shashi R, Biotechnology A: Medicinal uses of Adansonia digitata L.: An endangered tree species. J Pharm Sci Innov 2013; 2:14-16.

3. Suliman MB, Nour AH: Chemical Composition and Antibacterial Activity of Crude Extracts from Sudanese Medicinal Plant Adansonia digitata L. Chem Adv Mater 2017; 2.

4. Vermaak I, Kamatou GPP, Komane-Mofokeng B, Viljoen AM, Beckett K: African seed oils of commercial importance - Cosmetic applications. South African J Bot 2011; 77:920-933.

5. Nemarundwe N, Le Breton G, Chibaya I: Linking rural producers of natural products to global markets for poverty alleviation in southern Africa. XIII World For Congr Proc 2009.

6. Braca A, Sinisgalli C, De Leo M, Muscatello B, Cioni PL, Milella L, et al.: Phytochemical Profile, Antioxidant and Antidiabetic Activities of Adansonia digitata L. (Baobab) from Mali, as a Source of Health-Promoting Compounds. Molecules 2018; 23:3104.

7. Garvey R, Clegg M, Coe S: The acute effects of baobab fruit (Adansonia digitata) on satiety in healthy adults. Nutr Health 2017; 23:83-86.

8. Asero R, Mistrello G, Amato S: Detection of pan-allergens in commercial pollen extracts for allergen immunotherapy. Ann Allergy, Asthma Immunol 2016; 117:180-185.

9. Martini M, Swiontek K, Antonicelli L, Garritani MS, Bilò MB, Mistrello G, et al.: Lysozyme, a new allergen in donkey's milk. Clin Exp Allergy 2018; 48:1521-1523.

10. Grayson MH, Feldman S, Prince BT, Patel PJ, Matsui EC, Apter AJ: Advances in asthma in 2017: Mechanisms, biologics, and genetics. J Allergy Clin Immunol 2018; 142(5):1423-1436. 